\title{
DIMENSIONAL ACCURACY OF PARTS MANUFACTURED BY 3D PRINTING FOR INTERACTION IN VIRTUAL REALITY
}

\author{
Filip Górski' ${ }^{1}$ Radosław Wichniarek', Wiesław Kuczko' ${ }^{1}$ Przemysław Zawadzki' ${ }^{1}$ Paweł Buń1 \\ Poznan University of Technology, Chair of Production Engineering and Management, Piotrowo 3 St., 61-138 \\ Poznan, Poland, e-mail: filip.gorski@put.poznan.pl, wieslaw.kuczko@put.poznan.pl, radoslaw.wichniarek@ \\ put.poznan.pl, pawel.bun@put.poznan.pl, przemyslaw.zawadzki@put.poznan.pl
}

Received: 2017.10.24

Accepted: 2017.11.01

Published: 2017.12.05

\begin{abstract}
Realism of a Virtual Reality simulation can be improved by use of physical objects tracked in real time. The paper presents possibility of using low-cost FDM process, realized by MakerBot Replicator 2X machine, in comparison with a professional one to build tooling for a simulation of ultrasound examination procedure. The objects were manufactured on two different machines out of ABS material, 3D scanned for accuracy testing and finally possibilities of their use in a VR system were evaluated.
\end{abstract}

Keywords: 3D Printing, dimensional accuracy, Virtual Reality, ultrasound head.

\section{INTRODUCTION}

The Virtual Reality (VR) technology uses digitally built worlds to create a sense of presence at a user and help realize certain tasks faster and more effectively. As the technology develops and hardware prices drop, VR becomes more and more widely applied in entertainment, professional training [8] (especially regarding medicine [9]), in some industry branches [11], specialized learning and languages teaching [1], among other things.

Early VR-focused studies [10] prove that going into artificially prepared reality allows subconscious obtaining of competences in many fields. The state of being inside VR is known as the immersion. This phenomenon is used, among other things, for curing phobias [3], as well as in training. To obtain immersion, it is important to engage as many senses of a user as possible in interaction with a virtual environment. It is possible to achieve in many ways, among other things by representation of movement of real objects, manipulated by a user, in an application. The more real, physical object is similar to its virtual representation (both in terms of shape and movement accuracy), the easier is to achieve full immersion state, which translates into increase of training effectiveness [4]. The physical objects which can be tracked for manipulation in virtual environments are often manufactured using 3D printing techniques [7]. As in recent years many low-cost $3 \mathrm{D}$ printers were introduced to the market (usually working in Fused Deposition Modeling - FDM - technology), it has become more and more possible to produce additional objects tracked inside a virtual simulation due to greatly reduced costs and acceptable material strength [5]. The paper addresses the problem of effective manufacturing of objects aiding VR educational simulations using low-cost $3 \mathrm{D}$ printers.

\section{MATERIALS AND METHODS}

\section{Case and problem definition - medical VR application}

The simulation of ultrasound examination in VR is an expansion of an application created for Poznan University of Medical Sciences. According to initial assumptions, it was a virtual 3D human anatomy atlas, with the following functions: 
- Lecture - a scenario regarding a specific anatomical or physiological problem presented interactively by a teacher,

- Exercise - application used directly by students for manipulation of the virtual human model,

- Immersive presentations - single-person use, with application of a Head-Mounted Display (HMD).

The atlas was decided to be expanded with possibilities of training certain diagnostic procedures. The ultrasound examination was selected for the initial studies. The aim was to make the examination simulation realistic and inexpensive at the same time. Therefore, it was decided to build an additional device - a physical representation of the manual head for the ultrasound examination (in real life operated by a doctor who is performing the procedure), along with a physical phantom of a patient (in form of a mannequin). The device was manufactured using two different 3D printers, a low-cost 3D printer - MakerBot Replicator 2X, and a professional 3D printer - Dimension BST 1200. The aim of the studies presented in this paper was to compare both processes and evaluate if the device manufactured using a low-cost device can be successfully used in professional training of the ultrasound examination procedure using Virtual Reality.

\section{MANIPULATION IDEA - A TRACKING SYSTEM}

A tracking system is a device which allows real-time measurement of position and/or orientation of a given object. Usually tracked objects are special markers. There are certain devices, which allow tracking objects of any shape, by placing patterns of markers on them. This concept is utilized in the described ultrasound examination procedure, where a special object - a head for manipulation - is covered in markers recognized by a tracking system. The system used in the presented studies is PST-55 - it can track objects distant between $40 \mathrm{~cm}$ and several meters from the device, by means of infrared light detection. The device sends a wave of IR light, which is reflected back by the retroactive markers. If their pattern is recognized as forming a previously recorded object (shape), its position is calculated by analyzing image from two cameras built inside the device. It is important for the device to see at least 4 markers simultaneously, so the more markers on a surface of a given object, the better [2]. Figure 1 presents a general concept of markers and their recognition by the software.

Initial shape of the ultrasound examination device (see Fig. 1) was not properly recognized in the preliminary studies by the authors. That is why it was decided to change its geometry and expand it with additional elements for larger area to put markers into.

\section{ULTRASOUND HEAD MODELS PREPARATION}

The studied objects were models of heads used for ultrasound examination of human abdominal cavity. These models were prepared on the basis of commercial ultrasound examination systems. To make it more realistic, the physical objects were 3D scanned using the ATOS I optical scanner by the GOM company, with measure-

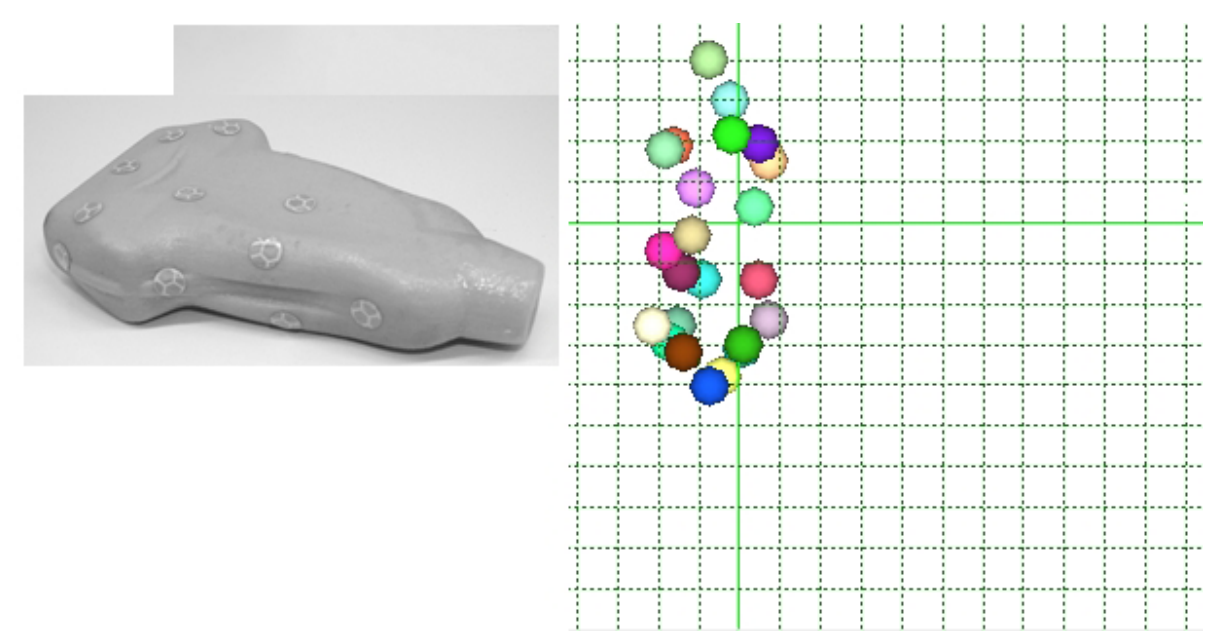

Fig. 1. Markers of the PST-55 system and their recognition in the system software [2] 
ment field of $125 \times 125 \mathrm{~mm}$. Three-dimensional scanning ensures rapid time of measurement and its result is a point cloud [6]. The initial head was re-created from the $3 \mathrm{D}$ scan and the modified models were prepared directly in the CATIA v5 CAD system, on the basis of 3D scans. The two models (Fig. 2) are based on real ultrasound devices, applied to examine different internal body areas (see Table 1 for details).

To use the re-created head in a VR simulation, a contact sensor was built in the head tip and the models were modified to allow its assembly. The contact sensor usually is non-present in real ultrasound examination head. In the real examination, the image of the patient's body internals is visible only when the head touches the skin. To obtain the same effect for the computer simulation in VR, it is necessary to detect a moment when user touches the physical phantom. The contact sensor is the easiest and least expensive method to ensure acceptable level of realism of simulation. The signal from the sensor can be easily accessed in available software, e.g. by emulating a mouse click.

Main part of the head was divided in 2 parts, which can be assembled using specially designed snap fasteners. The cable channel for the contact sensor was shaped in a way to not cause discomfort to the operator. Dividing the model directly along the channel axis allowed to avoid necessity of using support material in the Fused Deposition Modelling process. Figure 2 presents location of exit of the cable channel in both shapes of heads for the examination.

\section{MANUFACTURING OF ULTRASOUND HEADS}

In the previous work by the authors, modified head models manufactured by 3D Printing process were used. The 3D Printing was realized using ZPrinter 310 machine (3D Systems com- pany), out of a powder based on gypsum, joined by a binder based on methyl alcohol (old head visible in Fig. 1).

The new models of examination heads were manufactured using the Fused Deposition Modelling technology. The FDM technology consists in linear deposition of plasticized thermoplastic material, extruded by a nozzle of a small diameter, by a special head. The extrusion head can move in two axes (XY) and the table (on which the model is made) can move in the vertical ( $\mathrm{Z}$ ) axis. After each layer is made, the table goes down in the $\mathrm{Z}$ axis leaving a space equal to desired layer thickness between itself and a nozzle. To ensure support of geometry of manufactured object, if subsequent layer contour is significantly going beyond the previous layer, it is necessary to build special support structures. They are also made of thermoplastic material, but with different mechanical properties, which makes it possible to mechanically or chemically separate them from the actual object after the layer deposition is finished [11]. The head models were both manufactured using the Dimension BST 1200 - professional machine - and the MakerBot Replicator $2 \mathrm{X}-\mathrm{a}$ low-cost machine. Manufacturing parameters are presented in Table 1.

After manufacturing the heads, it was necessary to remove supports (Fig. 3). Relatively large difference between heads manufactured using two different machines is a result of applying two different materials. The HIPS (polystyrene) material used in the MakerBot Replicator $2 \mathrm{X}$ machine is removed in a much easier manner than the ABS material used in the Dimension BST 1200 machine. Moreover, it can be removed chemically (using citric acid) if the support is located in places difficult to reach with manual tools - it was not the case here, all the supports were removed mechanically.

To ensure proper fitting of parts of the head, proper assembly clearances were assumed

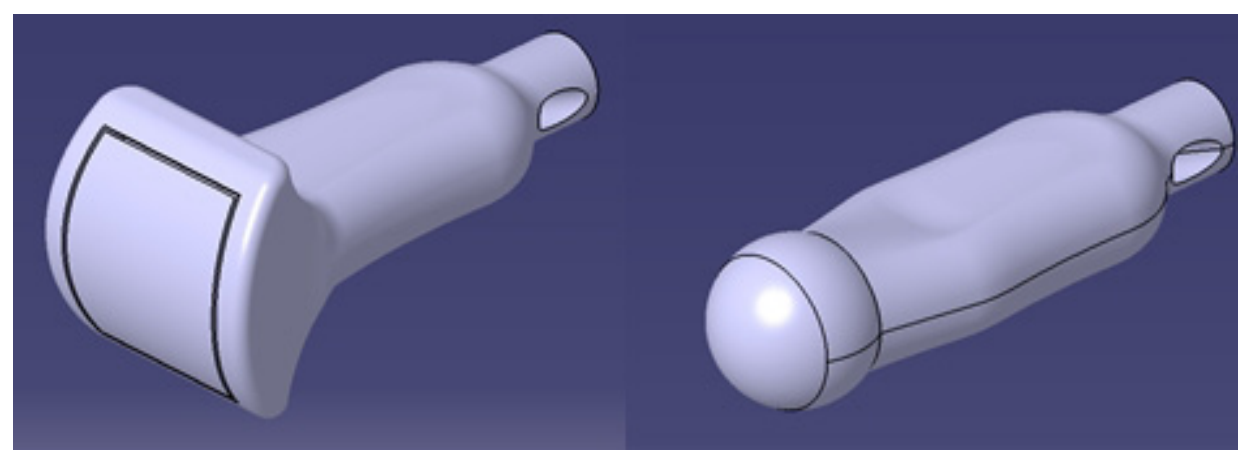

Fig. 2. Models of ultrasound examination heads with visible exit of the cable channel 
Table 1. Comparison of manufacturing parameters for heads produced using BST-1200 and MakerBot Replicator 2X machines

\begin{tabular}{|c|c|c|}
\hline Machine/ parameter & BST 1200 & Makerbot Replicator $2 X$ \\
\hline \multicolumn{3}{|c|}{ Parameters common for both heads } \\
\hline Infill & Sparse (linear) & Sparse (hexagonal) \\
\hline Layerthickness & $0.254 \mathrm{~mm}$ & $0.25 \mathrm{~mm}$ \\
\hline Model material & Dimension P400 black (ABS) & Makerbot True Black ABS \\
\hline Supportmaterial & Dimension P400-RP (ABS) & MakerbotDissolvableFilament (HIPS) \\
\hline \multirow[t]{3}{*}{ Temp. } & Model \&support : $300{ }^{\circ} \mathrm{C}$ & Model : $230^{\circ} \mathrm{C}$ \\
\hline & Chamber : $74{ }^{\circ} \mathrm{C}$ & Support : $250^{\circ} \mathrm{C}$ \\
\hline & & Table : $110^{\circ} \mathrm{C}$ \\
\hline Device preparationtime & $\begin{array}{l}1 \text { min (cleaning and assembly of } \\
\text { a tray) }\end{array}$ & $\begin{array}{l}7 \text { min (tablecleaning, kaptontapedeposition, coveringtape } \\
\text { with ABS dissolved in acetone for bettersticking) }\end{array}$ \\
\hline \multicolumn{3}{|c|}{ Wide head GE RSP6-16-RS 3D/4D Linear Probe } \\
\hline \multicolumn{3}{|c|}{ Applications: Small Parts, Vascular, Pediatrics, Ortho } \\
\hline Productiontime & $6 \mathrm{~h} 20 \mathrm{~min}$ & $5 \mathrm{~h}$ \\
\hline Model material & $75.6 \mathrm{~g}$ & $70 \mathrm{~g}$ (purgewallsused) \\
\hline Supportmaterial & $27.8 \mathrm{~g}$ & $24 \mathrm{~g}$ (purgewallsused) \\
\hline Supportremovaltime & $12 \min$ & $2 \min$ \\
\hline Approx. cost & 700 PLN & 250 PLN \\
\hline \multicolumn{3}{|c|}{ Sphericalhead GE RNA5-9 } \\
\hline \multicolumn{3}{|c|}{ Applications: Neonatal, Pediatrics } \\
\hline Productiontime & 3h $48 \mathrm{~min}$ & 3h $21 \mathrm{~min}$ \\
\hline Model material & $47.46 \mathrm{~g}$ & $47.4 \mathrm{~g}$ (purgewallsused) \\
\hline Supportmaterial & $19.74 \mathrm{~g}$ & $16 \mathrm{~g}$ (purgewallsused) \\
\hline Supportremovaltime & $7 \mathrm{~min}$ & $1 \mathrm{~min}$ \\
\hline Approx. cost & 450 PLN & 175 PLN \\
\hline
\end{tabular}
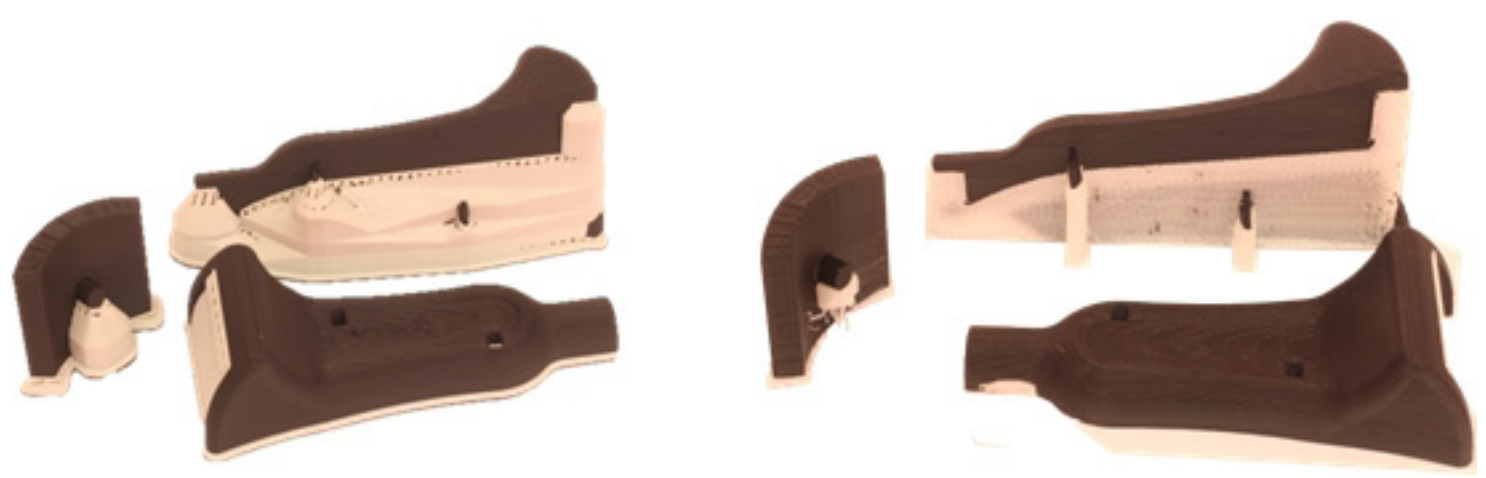

Fig. 3. Heads with support, left - BST 1200, right- MakerBot Replicator 2X

while designing the parts (presented in Fig. 4). Size of clearance was assumed $0.4 \mathrm{~mm}$ total (0.2 $\mathrm{mm}$ per one side).

Elements manufactured using the BST 1200 professional machine were possible to assembly directly after support removal. After assembly, there was a minimal clearance between the parts - assumed clearance size could be smaller, to ensure better snap fitting.
In case of the Replicator $2 \mathrm{X}$ machine, it was not possible to assembly both halves of the main head frame - there was no clearance. A layer of ABS material had to be removed on both sides of the snap fasteners, to enable joining the parts together $(0.5 \mathrm{~mm}$ total $)$. Removal of the material did not cause obtaining clearance - the joining was tight, although the two halves were not matched precisely, with a visible displacement. 


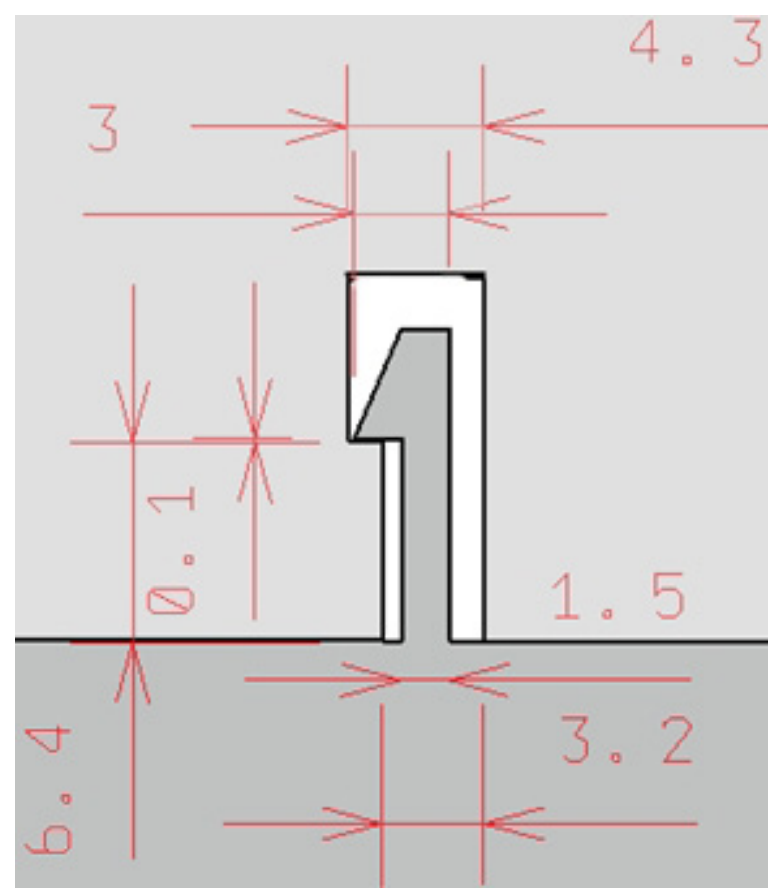

Fig. 4. Assumed assembly clearances

\section{RESULTS AND DISCUSSION}

The visual evaluation was performed first. In case of objects manufactured using the BST 1200 machine, the threads of material are visibly more parallel to each other, with no "waving" effect on side walls. The only visible defect is a "sew" perpendicular to layer division plane, caused by breaking material during layer contour deposition - it always occurs at the same point on the layer contour. The objects manufactured using the MakerBot Replicator 2X machine do not have the "sew", as it is possible to eliminate it by software means (starting and finishing the layer contour in each consecutive layer is shifted by a small distance). Still, visual quality of deposed threads in the low-cost machine was visibly worse than in the professional machine. Layers in contact with the support material were slightly displaced.

After initial visual evaluation, the parts were assembled together, to be used in the VR simulation. As the assembly process had different course for objects from each machine, as mentioned in the chapter 2.4, it was decided to investigate the obtained accuracy by 3D scanning. The manufactured wide heads were scanned using the GOM Atos Compact Scan 5M optical 3D scanner, using a measurement field of $150 \times 110 \times 110 \mathrm{~mm}$. Then, data analysis (matching scan with the nominal solid CAD model) was performed and colorful deviation maps were prepared. Selected maps are presented in Fig. 5 and 6.
Accuracy, approximated by an average deviation of the "best fit" method of matching between the CAD model and the scans, was 0.098 $\mathrm{mm}$ for the wide head manufactured by the BST 1200 machine and $0.147 \mathrm{~mm}$ for the Replicator $2 \mathrm{X}$ machine. For the BST 1200 machine, 100\% of measured points were in the $+/-0.5 \mathrm{~mm}$ tolerance field and $99.4 \%$ in $+/-0.38 \mathrm{~mm}$ field. For the Replicator $2 \mathrm{X}$ machine, the percentage for the same tolerance fields were $95.3 \%$ and $89.7 \%$, respectively.

It can be therefore assumed, that accuracy of the low-cost FDM process is far worse than the professional one, despite roughly the same parameters of manufacturing (leaving aside construction differences between two machines). The parts manufactured by the Replicator $2 \mathrm{X}$ machine require higher assembly clearances or manual processing after manufacturing to assure the proper assembly.

After the accuracy study, the heads were covered with markers of the PST-55 tracking system. Their arrangements were then introduced to the tracker's memory. Markers on the devices and their visibility in the tracker software are presented in Fig. 7.

The practical tests have proven that in case of both ultrasound examination heads, method of gripping has significant influence on detection of marker arrangement in the tracker software. The authors decided to solve this problem by adding extra geometrical elements on top of each head (Fig. 8), with additional markers, impossible to cover while gripping a head with one hand. The additional elements are universal and are assembled by snap fitting, but their addition requires recalibration of tracked marker arrangement. After expanding the heads with additional markers, recognition of devices by the tracker in the assumed tracking space increased significantly, to an acceptable level (objects visible and tracked more than $95 \%$ of manipulation time).

During the tests, the following observations were made regarding influence of manufacturing quality on use of objects in virtual simulation:

- Worse surface quality in objects from the Replicator 2X machine had negative influence on placement of markers of a tracking system. They are placed more easily and recognized more effectively when the surface is planar. No problems were observed for heads manufactured by the professional machine.

- Ergonomic quality of heads manufactured by the low-cost machine was slightly lower due to 


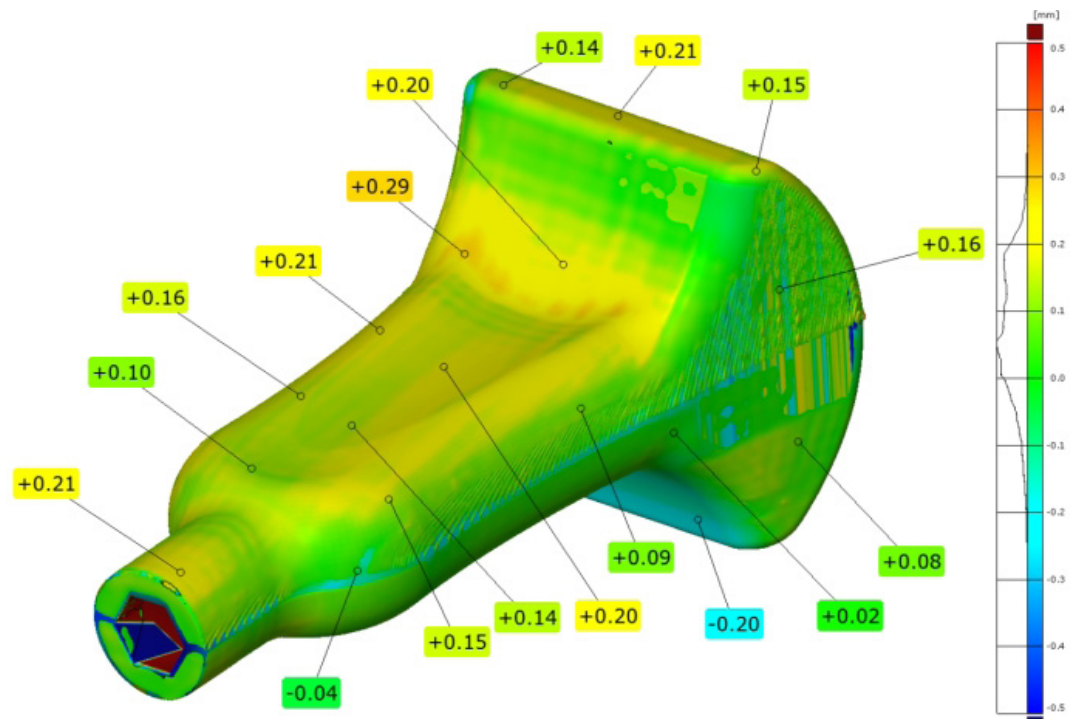

Fig. 5. Colorful deviation map for the wide head made using the Dimension BST 1200 machine, scale between -0.5 and $+0.5 \mathrm{~mm}$
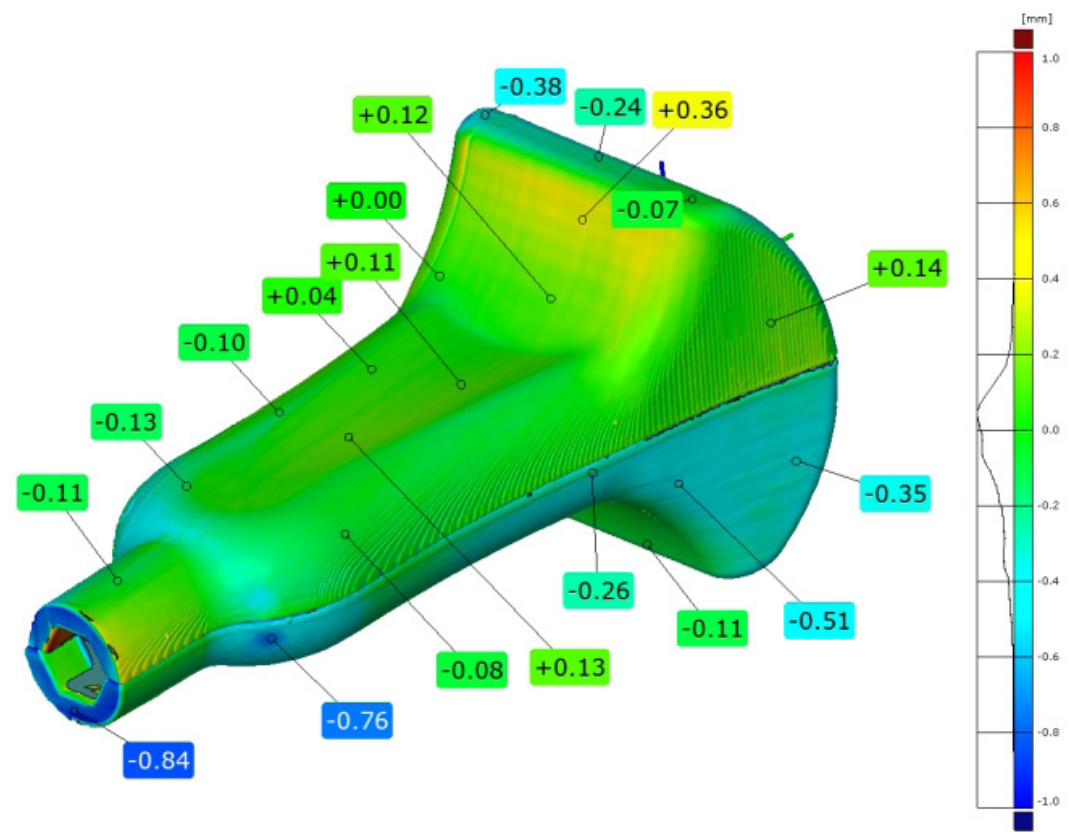

Fig. 6. Colorful deviation map for the wide head made using the MakerBot Replicator $2 X$ machine, scale between -1.0 and $+1.0 \mathrm{~mm}$
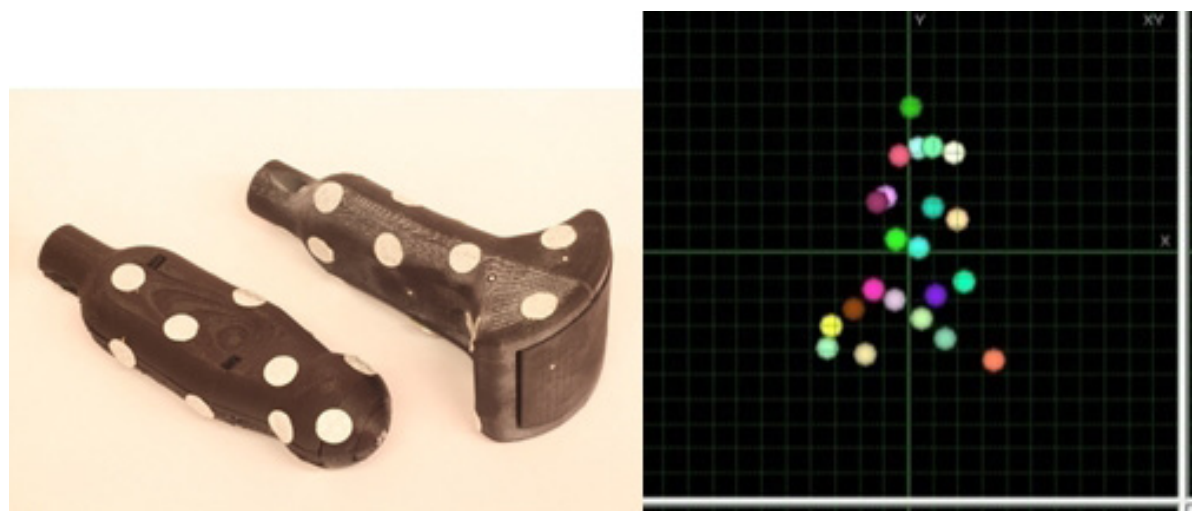

Fig. 7. Markers on 3D printed heads (left) and recognition of right head in in the tracker software for wide head (right) 

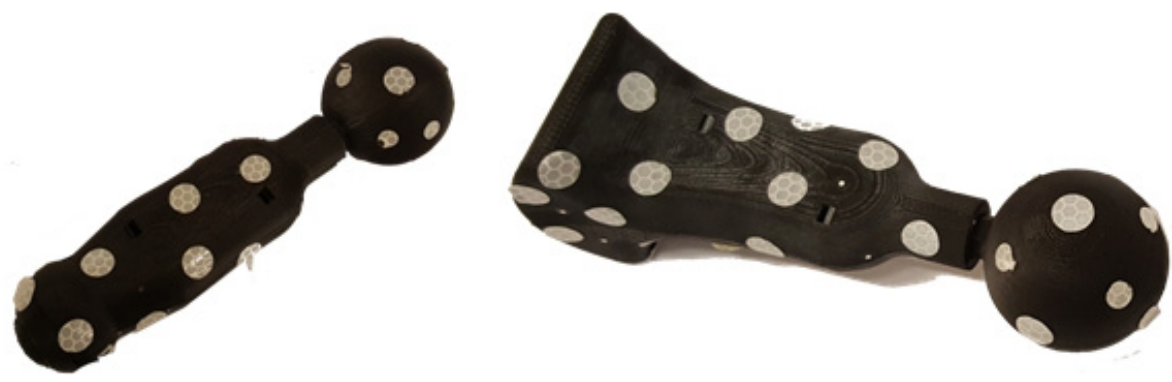

Fig. 8. Heads with additional 3D printed geometry, allowing better recognition by the PST-55 tracker

displacement of head parts and worse surface quality, but it was still on an acceptable level.

- The above mentioned flaws can be reduced if proper post-processing is applied, e.g. chemical and mechanical surface polishing.

- Despite the flaws and visible defects, it is still possible to effectively use the objects manufactured using a low-cost $3 \mathrm{D}$ printed in a training simulation.

\section{SUMMARY}

The performed processes and tests proved, that it is possible to effectively use low-cost 3D printers in professional VR education simulations. There is a number of problems with the low-cost process itself - the Replicator $2 \mathrm{X}$ machine must be supervised constantly as the nozzle is prone to clogging and breakage of material thread. Both situations require immediate action from the machine operator. The Replicator $2 \mathrm{X}$ (similarly to other low-cost FDM devices) also requires longer time of machine preparation, as it is not equipped in replaceable trays. Obtained quality and accuracy are also lower. Despite the defects, it is fully possible to obtain a working manipulator of dedicated, individualized shape for a simulation in Virtual Reality. The cost is significantly lower, therefore it is a recommended approach, although it requires higher qualifications of the machine operator.

It is noteworthy, that VR simulations can be successfully expanded with $3 \mathrm{D}$ printed accessories to further increase realism of simulations, which translates into better educational results. Both VR and $3 \mathrm{D}$ printing technologies have become more accessible in recent years due to emerging of lowcost processes, so the hybrid approach (joining virtual and physical objects) will become more and more widespread in authors' opinion.
Future work will consist in completing the VR simulation and conducting more tests with a group of students.

\section{REFERENCES}

1. Brzestrzynski W. Kształcenie odtwarzające środowisko immersyjne dla przyswajania języka obcego (,Education re-creating immersive environment for learning of a foreign language"), Neodidagmata 31/32, 2010/2011, 161-174.

2. Buń P., et al. Immersive educational simulation of medical ultrasound examination. Procedia Computer Science, 75, 2015, 186-194.

3. Buń P., et al. Low - Cost Devices Used in Virtual Reality Exposure Therapy. Procedia Computer Science, 104, 2017, 445-45.

4. Chua C. K., et al. Rapid Prototyping: Principles and Applications. World Scientific Publishing Co. Pte. Ltd., Singapore, 2010, 25-35.

5. Gajdoš I., et al. Structure and tensile properties evaluation of samples produced by Fused Deposition Modeling. Open Engineering, 1(6), 2016, 86-89.

6. Gessner A. and Staniek R. Evaluation of accuracy and reproducibility of the optical measuring system in cast machine tool body assessment. Advances in Manufacturing Science and Technology, 36(1), 2012, 65-72.

7. Górski F., et al. Integration of virtual reality and additive manufacturing technologies - hybrid approach to product development. Mechanik, 86(3), 2013, 173-176.

8. Grajewski D., et al. Immersive and Haptic Educational Simulations of Assembly Workplace Conditions. Procedia Computer Science, 75, 2015, 359-365.

9. Hamrol A., et al. Virtual 3D atlas of a human body - development of an educational medical software application. Procedia Computer Science, 25, 2013, 302-314.

10. Slater M., et al. Taking Steps: The Influence of a Walking Technique on Presence in Virtual Reality. ACM Transactions on Computer-Human Interaction (TOCHI), 2(3), 1995, 201-219.

11. We Y., et al. The Virtual Reality Applied in Construction Machinery Industry. Lecture Notes in Computer Science, 8022, 2013, 340-349. 